\title{
The nature of restructuring in insight: An individual-differences approach
}

\author{
IVAN K. ASH \\ Old Dominion University, Norfolk, Virginia \\ and \\ JENNIFER WILEY \\ University of Illinois, Chicago, Illinois
}

\begin{abstract}
The insightful problem-solving process has been proposed to involve three main phases: an initial representation phase, in which the solver inappropriately represents the problem; an initial search through the faulty problem space that may lead to impasse; and a postimpasse restructuring phase. Some theories propose that the restructuring phase involves controlled search processes, whereas other theories propose that restructuring is achieved through the automatic redistribution of activation in long-term memory. In this study, we used correlations between working memory (WM) span measures and problemsolving success to test the predictions of these different theories. One group of participants received a set of insight problems that allowed for a large initial faulty search space, whereas another group received a matched set that constrained the initial faulty search space in order to isolate the restructuring phase of the insightful process. The results suggest that increased ability to control attention (as measured by WM span tasks) predicts an individual's ability to successfully solve problems that involve both the initial search phase and the restructuring phase. However, individual differences in ability to control attention do not predict success on problems that isolate the restructuring phase. These results are interpreted as supporting an automatic-redistribution-of-activation account of restructuring.
\end{abstract}

Theories of problem solving generally include two main stages: representation and solution. The locus of difficulty in problem solving is typically attributed to the maintenance of goals, plans, and partial solutions as one navigates through the problem space and manipulates the elements of the representation in order to reach a solution. Many of the factors that cause difficulties in problem solving have been related to the construct of working memory (WM; Baddeley \& Hitch, 1974; Newell \& Simon, 1972). For example, Hitch (1978) found that mistakes in mental arithmetic increase as the number of intervening operations between partial and final solutions increase. In addition to length of solution path, difficulty varies with the breadth of the problem space and the number of constraints on the possible successful solution paths. In cases in which more information needs to be searched or main-

This research was supported by a grant from the UIC Campus Research Board and by National Science Foundation Grant 0347887 to the second author. Any opinions, findings, conclusions, or recommendations expressed in this material are those of the authors and do not necessarily reflect the views of either institution. We thank Greg Colflesh, Andy Conway, Melinda Jensen, Cara Jolly, Trina Kershaw, Tim Miura, Chris Sanchez, and undergraduate research assistants Mital Patel, Olivia Jedlicki, and Pervin Nadzaku for their assistance and input on this project. We thank Colleen Seifert for her comments on a previous version and her suggestion of an interesting alternative explanation for our results. Correspondence concerning this article should be addressed to I. K. Ash, Department of Psychology, Old Dominion University, Norfolk, VA 23529-0267 (e-mail: iash@odu.edu). tained, problems become more difficult. Hence, people's limited ability to devote attentional resources to multiple simultaneous memory and computation processes can be seen as one of the primary obstacles in problem solving. This leads to the prediction that differences in the ability to actively control attentional resources should relate to the ability to successfully search a problem space and reach a solution (Hambrick \& Engle, 2003).

A special case of problem solving occurs when problems are unfamiliar and misleading (called insight problems). In these cases, solvers begin by forming a representation. However, prior experience often leads to an inappropriate representation that cannot lead to a correct solution. Nevertheless, the solver moves to a solution phase. Since the solution cannot be found with this initial representation, the solver will fail to reach a solution and may arrive at a point at which no further progress can be made, called an impasse. Therefore, on these types of problems, difficulty stems from two distinct obstacles. The first obstacle lies in the initial solution phase, where solvers must exhaust the operations available in the initial faulty problem space to reach impasse. However, these problems also present a second obstacle: the inappropriate operations and strategies activated by their faulty problem representation. Many researchers have proposed that this representational obstacle is resolved through processes that replace or change the initial faulty representation into a problem representation that allows the solver to search the appropriate problem space. Thus, a third stage, often referred 
to as restructuring, is important in successful insightful problem solving.

The processes that underlie restructuring are still unknown, with different researchers proposing different mechanisms. Some researchers have suggested that the mechanisms that lead to postimpasse restructuring are conscious, controlled, and attention-demanding processes (e.g., Davidson, 1995; Kaplan \& Simon, 1990). Alternatively, there are explanations of restructuring that rely on automatic processes, such as the automatic spread of activation through the semantic networks of long-term memory (Knoblich, Ohlsson, Haider, \& Rhenius, 1999; Ohlsson, 1992; Seifert, Meyer, Davidson, Patalano, \& Yaniv, 1995).

In order to investigate the nature of the restructuring process, we employed an individual-differences approach and tested for the relationship between insight problemsolving success and WM span measures. Research has suggested that individual differences in WM span tasks relate to individual differences in the central executive processes that allow individuals to actively control attentional resources (Kane, Bleckley, Conway, \& Engle, 2001). Individual differences in WM span measures reliably predict performance on tasks that involve resolution of interference, suppression of a habitual response, goal maintenance, and response competition, all of which are relevant for problem solving via controlled search strategies. On the other hand, WM span measures do not reliably predict performance on tasks that involve habitual or overlearned responses or depend on the automatic spread of activation (see, e.g., Conway \& Engle, 1994; Kane \& Engle, 2003). Therefore, this body of research suggests that WM span measures predict performance on cognitive tasks because they are sensitive to individual differences in people's ability to actively control and allocate attentional resources and not because they measure the "size" of an individual's immediate memory buffers (see Conway $\&$ Kane, 2001, for a review). Therefore, we propose that the correlation between WM span scores and success at the restructuring stage of the insightful problem-solving process can be used to test the predictions of different accounts of restructuring. In order to test this hypothesis, one must be able to separate the processes involved in overcoming the obstacles that relate to the initial search and exhaustion of the faulty problem space from the processes involved in overcoming the second representation obstacle.

In the present study, we created two variants of a set of insight problems. One version of the problems was designed so that success would depend on the entire insightful process (initial search and postimpasse restructuring). The second version of each problem was designed to reduce the size of the initial faulty search space, thereby comparatively isolating the postimpasse restructuring phase of the insightful process. This manipulation was inspired by Ormerod, MacGregor, and Chronicle (2002), who developed an insight problem in which the number of moves available in the faulty solution space could be manipulated without changing the proposed representational difficulty or the insightful solution. The two versions of the coin problem they developed are presented in Figure 1 (Problem 1). Ormerod et al. suggested that individuals initially try to solve these problems by moving coins around on the two-dimensional plane. They proposed that the primary insight in this problem involves switching from a representation where move is defined as a twodimensional change in position to a representation that allows for three-dimensional moves, such as stacking. Note that in the few moves available (FMA) variant, no possible two-dimensional move makes any coin touch three other coins. On the other hand, in the many moves available (MMA) variant, there are 20 available two-dimensional moves in which a coin can touch three other coins. However, none of these two-dimensional moves leads to all the coins' touching three other coins. Only stacking the coins, as shown in the solution in Figure 1, will lead to the correct configuration.

If one thinks about the stages of insight problem solving proposed earlier (schematically diagrammed in Figure 2), one can see that solving the MMA variant of the coin problem, which has multiple potential two-dimensional moves that partially satisfy the goal of the problem, would rely heavily on one's ability to successfully exhaust the faulty problem representation. However, the FMA variant of the coin problem has no moves available in the initial faulty representation. This effectively removes the search through the initial faulty representation (the second box in Figure 2) from the problem-solving process, and solvers can begin at or near a state of impasse. Therefore, only the processes involved in resolving impasse or restructuring would play an important role in solving this version of the problem.

In this study, we created matched sets of problems that varied in the size of the faulty initial search space on the basis of Ormerod et al.'s (2002) manipulation. To do this, one must first identify a problem's proposed representational obstacle (i.e., the incorrect operations suggested by the theoretical faulty representation). Next, one must manipulate the structure of the problem in a way that affords a greater number of or fewer different inappropriate operations or moves, without changing the proposed insight or representational change that can lead to the correct solution. Figure 1 presents the six classic insight problems for which we were able to devise FMA and MMA problem variants.

Manipulating the availability of potential inappropriate moves and, thereby, the size of the initial faulty search space allowed us to test the competing predictions of different accounts of the nature of the mechanisms involved in restructuring. If the controlled search theories of restructuring are correct, we would predict that WM span measures should be positively related to solution success on all problems, because higher capacity to control attention should lead to better performance at both the initial search and the restructuring phases. However, if the automatic activation accounts of restructuring are correct, we would predict an interaction of WM span and the number of moves in the initial faulty problem space on problem- 
Problems:

1) Coin Problem (from Ormerod, et al , 2002).

There are 8 coins in this picture. Move 2 coins so that each coin touches exactly 3 other coins. The coins will need to be separated into two groups.

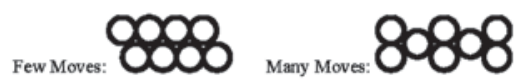

2) Glasses Problem (adapted from Ashcraft, 1994)

The picture below is of 6 glasses and 8 (or 13) coasters. The first 3 glasses contain liquid. Describe how you could make it so no 2 glasses containing liquid are next to each other and no 2 empty glasse are next to each other, while keeping 3 of the 6 glasses full. To do this, you are only allowed to move 1 glass and all glasses must end up resting on coasters.

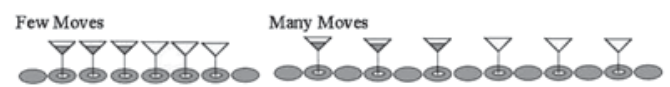

3) Matchstick Operator Problem (based on Knoblich et al., 1999)

The matchsticks in the following problem make Roman numerals. Notice that both sides of the equation are no equal. You need to make these into correct arithmetic equations by moving only $c$ single match in each problem. The specific rules are:

A) only one matchstick is to be moved

B) a matchstick cannot be discarded; that is, it can only be moved from one position in the equation to another

C) an upright stick cannot count as a slanted stick, so
D) the result must be a correct arithmetic equation Move one matchstick to make this into a true equation:

Few Moves $X+I V=V \quad$ Many Moves $X I ! !+!=X !$

4) Matchstick Numeral Problem (Same instructions as Problem 3).

$$
{ }_{\text {Few Moves }} \mathrm{V}=\mathrm{XI}-1 \quad \text { Many Moves } V ! I=X ! I !-!
$$

5) Katona Squares (Katona, 1940)

Few Moves

Move 3 of the gray matchsticks to make 5 squares.

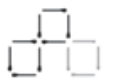

Many Moves

Move 3 matchsticks to make 5 squares.

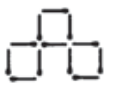

6) Triangles

Few Moves

Move the 3 gray matchsticks to make 5 equilateral triangles.

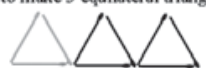

Many Moves

Move 3 matchsticks to make 5 equilateral

triangles.

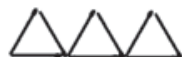

Solutions and Explanations of Problem Manipulation:

1) The insight is that the coins must be stacked into 2 three-dimensional piles. The solution to both versions appears to the right. In the MMA version there are 4 possible locations where a coin can be moved in the 2D array that allow it to touch 3 other coins, however there is no 2D arrangement that allows for all coins to be touching 3 other coins. On the FMA version there is no possible location to move a coin in the $2 \mathrm{D}$ array that will let it touch 3 other coins.

2) The insight is that "move" does not only mean putting a glass in a new location but can mean picking up a full glass, emptying the liquid into an empty glass, and returning it to its original position. The solution presented here works for both versions. The FMA problem was constrained by placing the 6 glasses on coasters, with an extra coaster at either end, and adding the rule that all glasses must end up on coasters. This limited the potential destinations to 2 . In the MMA condition, coasters were placed between the glasses, increasing the number of possible move locations to 9 .

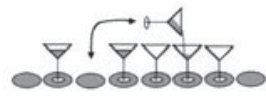

3) Solvers inappropriately assume that these problems should be solved by moving single matchsticks that represent the Roman numeral for "one" (I) to different locations in the problem. The insight is that solvers must move either a matchstick that is part of an operator (+ into -) or a matchstick that is part of a Roman numeral ( $\mathrm{X}$ into V). The MMA versions of these problems contained more single matchsticks representing Is than the FMA versions, thereby allowing for more possible solution paths that involve the movement of the sticks representing I's in the MMA versions.

Both operator problems require changing the + into -, and moving the vertical stick from the + to Rom

$X-|V=V I|$

4) The numeral problems require changing the $X$ to a $V$ or vice versa. The change $X$ answer is shown for the FMA problem and the change $V$ answer is shown for the MMA problem but both can problems can be solved using either method.

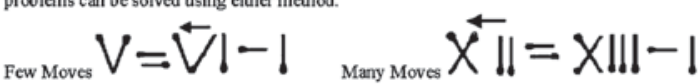

$5 \&$ ) The insight for both the Katona Squares and Triangles problems is that when four smaller triangles or squares are constructed, their outer edge also forms 1 larger triangle or square. The original MMA versions of these problems contain 9 sticks in the triangle problem and 12 sticks in the square problem. Presumably any combination of three sticks could be moved anywhere in different attempts at solving this problem. This results in a large number of initial available moves. In order to constrain the number of moves in the FMA condition, the three matchsticks that need to be moved in order to solve the problem were highlighted in a different color, and the directions stated that only
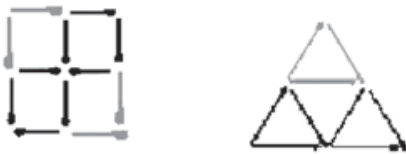

Figure 1. Many moves available (MMA) and few moves available (FMA) problem stimuli, with manipulation and solution explanations.

solving performance. According to these theories, the automatic processes involved in restructuring should be unrelated to individual differences in the capacity to control attention. Therefore, if restructuring is due to an automatic process, such as the passive spread of activation in long-term memory, we would predict no relationship between WM span measures and problem-solving success on the problems in which the restructuring phase has been isolated (i.e., on problems with few available moves in the initial faulty problem space). The automatic account of restructuring also predicts that success on problems with large initial problem spaces should correlate well with WM span measures, because higher capacity to control attention should be helpful in navigating the initial space to reach impasse.

\section{METHOD}

\section{Participants}

Two hundred seven University of Illinois at Chicago introductory psychology students participated as part of a course requirement. Three participants were dropped because of incomplete data, and 1 was dropped for having solution times above the maximum limit.

\section{Materials}

Reading Span Test (RSPAN) and Operation Span Test (OSPAN). The span task materials and procedures were taken directly from Kane et al. (2004). The span tasks require participants to remember target items while performing a concurrent reading comprehension or arithmetic task. The number of targets in a trial set varied between two and five, with three trials at each size.

Insight problems. Following Ormerod et al. (2002), we constructed six insight problems with MMA and FMA variants (Figure 1). The variants included Ormerod et al.'s (2002) coin problems, two sets of the matchstick arithmetic problems (Knoblich et al., 1999), Katona's (1940) matchstick squares and triangles problems, and the six glasses problem (Ashcraft, 1994). The problems were presented in packets that contained either all FMA problems or all MMA problems. The order of the problems was counterbalanced, resulting in six problem orders. Each packet began with a directions page, which explained the procedure and how to record times. The first problem in all the conditions was a noninsight matchstick arithmetic problem (Knoblich, Ohlsson, \& Raney, 2001). Then each of the six experimental problems followed. Finally, a two-page problem familiarity questionnaire asked the participants whether they had seen, solved, or remembered the answer to the each problem.

\section{Procedure}

The participants were first run through the WM span tasks (the WM phase) and then were randomly assigned to a problem type con- 


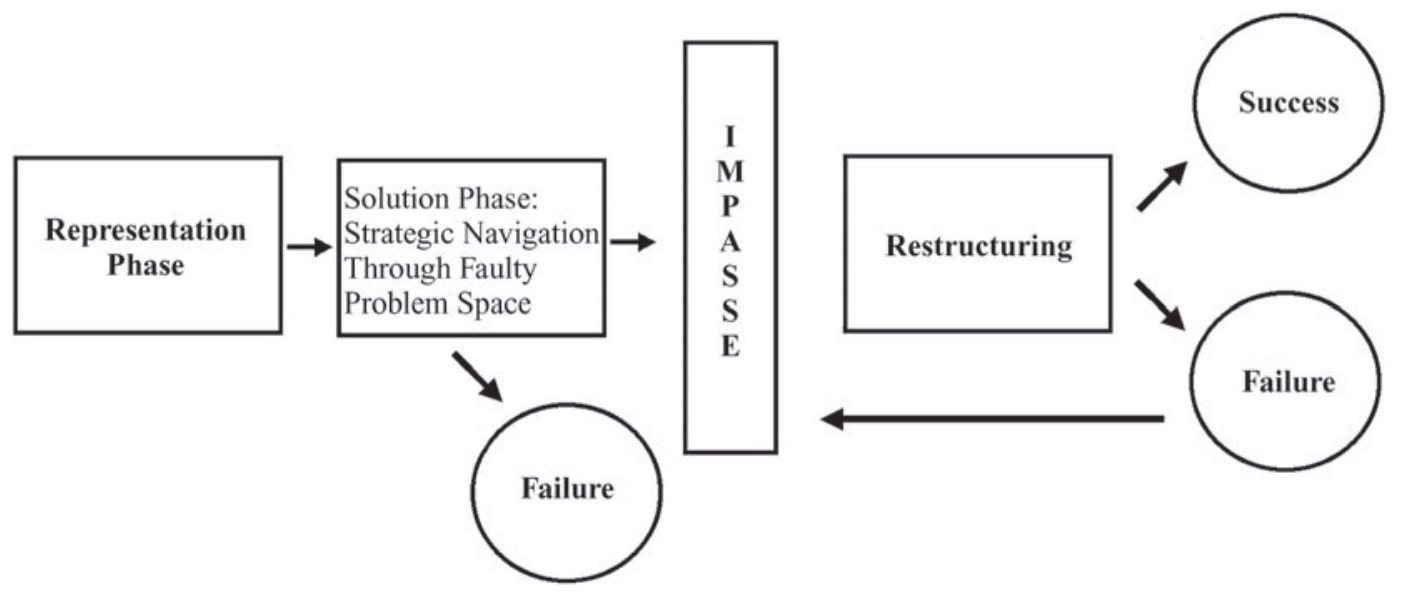

Figure 2. Flow chart representing the stages of insightful problem solving.

dition (MMA or FMA) and to one of six problem orders. Some of the participants completed the WM phase individually in one session and returned at a later time in the semester to complete the problemsolving phase in a classroom with other participants (two sessions). However, others completed the WM and problem-solving phases individually in a single 1-h session (one session). Besides these differences, all other aspects of the procedure were held constant.

Working memory phase. All the participants were run through the WM phase individually. This phase lasted approximately $30 \mathrm{~min}$. A participant's span score on each task was his or her average proportion of correct serial recall of targets across the different set sizes (RSPAN, $M=66 \%, S D=14$; OSPAN, $M=66 \%, S D=14 ; r=$ $.64, p<.001, N=203$ ). A WM span composite score (WMSPAN) was calculated by averaging the standardized RSPAN and OSPAN scores.

Problem-solving phase. The participants in the two-session group $(n=119)$ completed this phase in groups of 10 to 20 within the same semester as the WM phase. The participants in the onesession group $(n=87)$ completed this phase individually, directly after the WM phase.

The participants were informed that they would be performing a series of problem-solving tasks. They were told that they would be given 4 min to complete each problem. A large digital clock was placed in the front of the room. They were asked to write down the exact time, to the second, that they began each problem. If they finished before time had expired, they were asked not to begin the next problem. Instead, they were asked to write down the exact time they had finished the problem and to wait for the experimenter's signal to start the next problem. After completing the problems, the participants completed the problem familiarity questionnaire.

Solutions to the problems that did not violate the instructions of the problem were coded as correct. Any other answers or failures to answer were coded as incorrect. The starting and finishing times recorded by the participants were used to calculate solving times. The main indices of problem-solving performance were the total number of problems correctly solved ( $0-6)$ and the participant's average solving time on correctly solved problems. Questionnaires were coded as to how many of the problems the participants had seen before.

\section{RESULTS}

Theories of insightful problem solving propose that restructuring processes should occur specifically on unfamiliar problems. Some researchers have chosen to drop problems that participants were familiar with (e.g., Met- calfe \& Wiebe, 1987). However, since we could create only a limited number of stimulus items and the number of problems solved was a primary dependent variable, these factors made dropping single problems from analysis problematic. Therefore, consistent with prior research (e.g., Schooler, Ohlsson, \& Brooks, 1993), participants were dropped from analysis if they reported having seen one or more of the problems before. This resulted in 115 participants who were unfamiliar with all the target problems. All subsequent analyses were conducted on this sample, to control for prior problem knowledge.

\section{Descriptive Statistics}

Table 1 presents the numbers, ranges, means, standard deviations, skew, kurtosis, and reliability on the main variables in this study (composite WMSPAN score, total number of correctly solved problems, and average correct solving time). The skew and kurtosis values on all measures were within the recommended range for regression analysis (Kline, 1998). Furthermore, the range, standard deviations, and reliability estimates suggest similar spreads of distributions and reliability of measures across problem type groups. Finally, a series of 2 (one vs. two sessions) $\times 2$ (FMA vs. MMA) ANOVAs were conducted on WMSPAN, the total number of correctly solved problems, and the average correct solving time, in order to investigate whether the participants in the these groups differed on any of these variables. No significant main effects or interactions were detected on any of the variables. This suggests that any differences between the relationship of WM span and the indices of problem-solving success detected in the regression analysis are not due to between-group differences on any of the variables.

\section{Relation Between Working Memory Span and Insight Success}

In order to investigate the effect of the number of initial moves available in a problem space on the relationship between WM span scores and insightful problem-solving success (number of correctly solved problems and average 
Table 1

Descriptive Statistics for Working Memory Span (WMSPAN) Scores, Number of Correctly Solved Problems (Maximum of 6), and Average Solving Time (in Seconds; 240-sec Maximum) as a Function of Problem and Session Type Groups

\begin{tabular}{|c|c|c|c|c|c|c|c|c|}
\hline Session Type & Measure & $N$ & Range & $M$ & $S D$ & Skew & Kurtosis & Reliability \\
\hline \multicolumn{9}{|c|}{ FMA Problems } \\
\hline \multirow[t]{3}{*}{ Two sessions } & Total correct & 35 & 5.00 & 2.63 & 1.44 & -0.31 & -0.93 & \\
\hline & Average solving time & 32 & 204.33 & 109.90 & 45.72 & 0.54 & 0.94 & \\
\hline & WMSPAN & 35 & 3.78 & 0.16 & 0.88 & -0.03 & -0.31 & \\
\hline \multirow[t]{3}{*}{ One session } & Total correct & 26 & 3.00 & 2.46 & 1.07 & 0.00 & -1.18 & \\
\hline & Average solving time & 26 & 177.00 & 102.62 & 50.32 & 0.64 & -0.22 & \\
\hline & WMSPAN & 26 & 3.90 & -0.16 & 1.14 & -0.15 & -1.07 & \\
\hline \multirow[t]{3}{*}{ FMA total } & Total correct & 61 & 5.00 & 2.56 & 1.28 & -0.18 & -0.88 & $.56^{\mathrm{a}}$ \\
\hline & Average solving time & 58 & 204.33 & 106.64 & 47.54 & 0.55 & 0.17 & $\mathrm{NA}^{\mathrm{b}}$ \\
\hline & WMSPAN & 61 & 4.09 & 0.02 & 1.00 & -0.22 & -0.59 & $.83^{\mathrm{a}}$ \\
\hline \multicolumn{9}{|c|}{ MMA Problems } \\
\hline \multirow[t]{3}{*}{ Two sessions } & Total correct & 38 & 6.00 & 2.37 & 1.73 & 0.22 & -0.87 & \\
\hline & Average solving time & 30 & 198.50 & 112.49 & 51.36 & 1.46 & 1.83 & \\
\hline & WMSPAN & 38 & 2.79 & -0.07 & 0.67 & 0.11 & -0.40 & \\
\hline \multirow[t]{3}{*}{ One session } & Total correct & 16 & 5.00 & 2.13 & 1.75 & 0.56 & -0.77 & \\
\hline & Average solving time & 13 & 153.50 & 95.56 & 47.49 & 0.52 & -0.44 & \\
\hline & WMSPAN & 16 & 3.66 & -0.17 & 0.86 & -0.15 & 1.10 & \\
\hline \multirow[t]{3}{*}{ MMA total } & Total correct & 54 & 6.00 & 2.30 & 1.72 & 0.30 & -0.91 & $.51^{\mathrm{a}}$ \\
\hline & Average solving time & 43 & 205.00 & 107.37 & 50.27 & 1.20 & 1.47 & $\mathrm{NA}^{\mathrm{b}}$ \\
\hline & WMSPAN & 54 & 3.66 & -0.10 & 0.72 & -0.05 & 0.31 & $.63^{\mathrm{a}}$ \\
\hline
\end{tabular}

Note-FMA, few moves available; MMA, many moves available. aReliability calculated using Cronbach's alpha. bDifferent participants had different numbers of problems contribute to their average correct solving time, with some participants correctly solving only one problem. Therefore, it is impossible to calculate reliability, because that would require multiple measures on all subjects. However, a test for the homogeneity of variance on this measure showed no evidence that the groups differed in overall variability (Levene's test of homogeneity of variance, $F<1, p=.90)$.

correct solving time), we conducted two hierarchical multiple regressions. In the first block, the subjects' WMSPAN scores and dummy-coded problem type conditions were entered into the equation. In the second block, a WMSPAN $\times$ problem type interaction term was entered into the equation. This term was the product of the centered WMSPAN scores and the problem type dummy code.

The results of the regression on number of problems correctly solved are presented in Table 2 . The first block, containing problem type and WMSPAN, significantly predicted number of correctly solved problems $\left[R^{2}=.08 ; F(2,112)=\right.$ $4.89, p<.01]$. The results revealed that the higher a person's WMSPAN score, the more problems he or she was likely to solve correctly $(\beta=.27, p<.01)$. There was no effect of the number of available moves on number of correctly solved problems. The second block, in which the WMSPAN $\times$ problem type interaction was entered, resulted in a significant improvement in prediction $\left[\Delta R^{2}=.046 ; F(1,111)=\right.$ $5.81, p<.05]$. Figure 3 presents the scatterplot and regression lines for the relationship between WMSPAN and solution success for the FMA and MMA groups. On the MMA problems, there is a clear positive relationship between WMSPAN and number of problems solved. However, on the FMA problems, this relationship is attenuated.

The results of the regression on average correct solving time are presented in Table 3. Note that only the participants who solved at least one problem correctly could be used in this analysis, thereby reducing $N$ to 101 . The first model, containing problem type and WMSPAN, showed a trend toward predicting average solving time $\left[R^{2}=.05 ; F(2,98)=\right.$

Table 2

Results of the Hierarchical Multiple Regression on Number of Problems Correctly Solved

\begin{tabular}{clrrrrrr}
\hline Block & \multicolumn{1}{c}{ Term } & \multicolumn{1}{c}{$B$} & \multicolumn{1}{c}{$S E$} & $\beta$ & \multicolumn{1}{c}{$t$} & $p$ & $R^{2}$ \\
\hline \multirow{2}{*}{1} & Problem type & -0.204 & 0.273 & -.068 & -0.747 & .456 & .080 \\
& WMSPAN & 0.463 & 0.155 & .270 & 2.977 & .002 & \\
\multirow{2}{*}{2} & 2.547 & 0.186 & & & & \\
& Constant & -0.435 & 0.284 & -.145 & -1.534 & .128 & .126 \\
& Problem type & 0.214 & 0.184 & .125 & 1.163 & .247 & \\
& WMSPAN & 0.790 & 0.328 & .267 & 2.411 & .018 & \\
& WMSPAN $\times$ problem type & 2.553 & 0.183 & & & & \\
\hline
\end{tabular}

Note-Problem type, few moves available versus many moves available; WMSPAN, working memory span. 


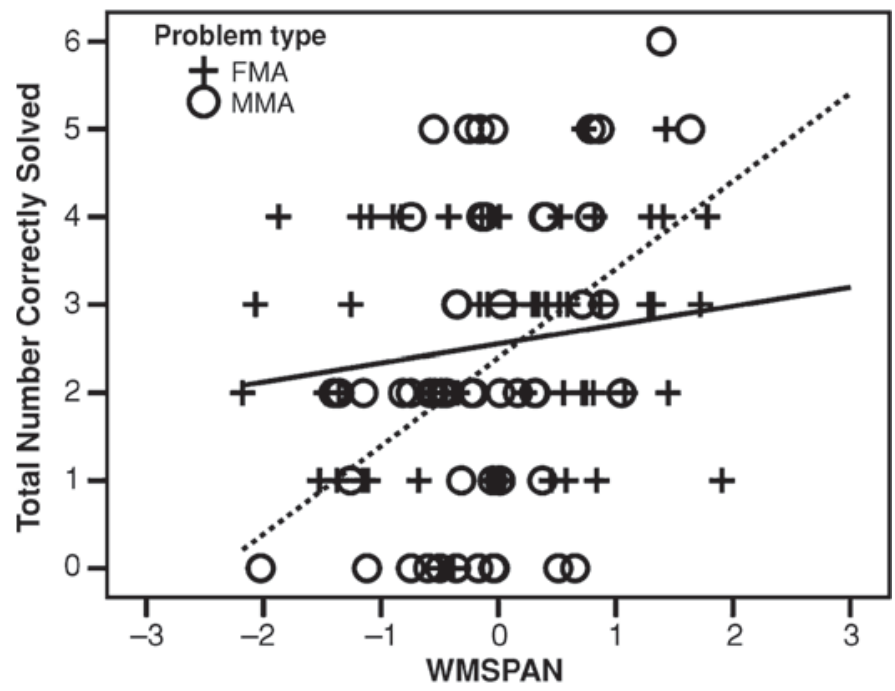

Figure 3. Scatterplot of the relationship of working memory span (WMSPAN) scores and number of problems correctly solved as a function of the number of moves available in the initial problem space. The dashed line represents the regression line for the many moves available (MMA) problems, and the solid line represents the regression line for the few moves available (FMA) problems.

$2.57, p=.07]$. Once again, WMSPAN was a significant predictor of solving success. Those with higher WM spans took less time to successfully solve problems $(\beta=-.22$, $p<.05$ ). However, there was no effect of the problem type on solving time. Although the introduction of the interaction term did not result in a significant improvement of fit, there was some evidence of an interaction trend that was similar to the one observed on the number of correctly solved problems variable $\left[\Delta R^{2}=.02 ; F(1,97)=2.06, p=.16\right]$. Figure 4 presents the scatterplot and regression lines for the relationship between WMSPAN and average solving time for the FMA and MMA groups. On the MMA problems, those with higher WMSPAN scores appear to have solved the problems more quickly. However, this relationship appears to be attenuated on the FMA problems.

\section{DISCUSSION}

Successfully solving unfamiliar and misleading problems is a multistage process involving an initial search through a faulty problem space that can lead to impasse and a postimpasse stage that involves restructuring the initial problem representation. The present experiment was performed to test between two explanations of restructuring: one based on controlled search processes and one based on the automatic redistribution of activation. Because prior research on WM span tasks had demonstrated that these scores predict performance only on tasks that place demands on central executive functioning and require controlled attention (Kane et al., 2001), we used correlations with WM span and problem-solving performance to attempt to discriminate between controlled and automatic accounts of restructuring.

In the present study, we explicitly took the different stages of insightful problem solving into account as we attempted to investigate the nature of restructuring. By decreasing the number of moves available in the initial problem representation, we created a condition (FMA) in which problem-solving success would be dependent primarily on the restructuring phase. This allowed us to

Table 3

Results of the Hierarchical Multiple Regression on Average Solving Time

\begin{tabular}{clrrrrrr}
\hline Block & \multicolumn{1}{c}{ Term } & \multicolumn{1}{c}{$S$} & \multicolumn{1}{c}{$S E$} & $\beta$ & \multicolumn{1}{c}{$t$} & $p$ & $R^{2}$ \\
\hline 1 & Problem type & -0.111 & 9.613 & -.001 & -0.012 & .991 & .05 \\
& WMSPAN & -12.085 & 5.329 & -.223 & -2.268 & .026 & \\
& Constant & 107.192 & 6.272 & & & & \\
2 & Problem type & 5.942 & 10.453 & .061 & 0.569 & .571 & .07 \\
& WMSPAN & -7.591 & 6.159 & -.140 & -1.233 & .221 & \\
& WMSPAN $\times$ problem type & -17.341 & 12.097 & -.173 & -1.433 & .155 & \\
& Constant & 106.985 & 6.241 & & & & \\
\hline
\end{tabular}

Note-Problem type, few moves available versus many moves available; WMSPAN, working memory span. 


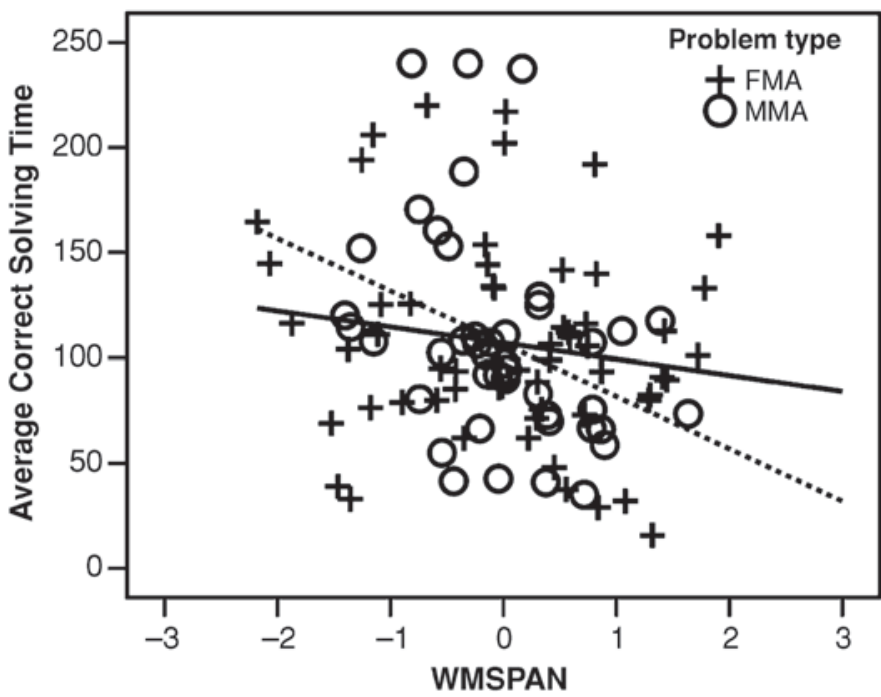

Figure 4. Scatterplot of the relationship of working memory span (WMSPAN) scores and average correct solving time as a function of the number of moves available in the initial problem space. The dashed line represents the regression line for the many moves available (MMA) problems, and the solid line represents the regression line for the few moves available (FMA) problems.

contrast success at the restructuring phase with success that required all phases of problem solving (which was the case with the MMA problems). We then correlated WM span scores with problem-solving success in each condition.

Although all theories of restructuring suggested that WM span measures should predict performance on the MMA problems, only the automatic restructuring theories of insight predicted that WM would predict solution success on the MMA problems but not on the FMA problems. This hypothesis was supported with both measures of problem-solving success. Individual differences in WM span measure predicted success only on the MMA problems. One possible explanation for this finding is that the FMA problems were so easy that they did not tax the attentional capacity of solvers. However, performance on the FMA problems was not at ceiling. The overall difficulty and amount of reliable variance was similar across MMA and FMA problems, but individual differences in the ability to control attention did not explain this variance for the FMA problems. Therefore, this particular result is consistent with the predictions of automatic theories of restructuring and is inconsistent with controlled search theories of restructuring.

An interesting and unanticipated finding from this study was that individuals with higher spans seemed to be more likely to succeed on the MMA than on the FMA problems, whereas individuals with lower spans seemed less likely to succeed on the MMA than on the FMA problems. This suggests the compelling hypothesis that, in some problem situations, increased attentional ability might facilitate restructuring processes. In particular, if the solver has the ability to maintain different failed solution attempts in memory, he or she may be more likely to recognize invariants in his or her failures. This might suggest that individuals with better control of attention are successfully employing processes such as those proposed by Kaplan and Simon (1990), but only in problem situations in which the initial faulty problem space allows for this type of exploration. This is an interesting possibility that could have been uncovered only with the present approach, which explicitly took into account the different stages of the insightful problem-solving process and individual differences in ability.

These results clearly show there is much to learn about the nature of the processes involved in insightful problem solving and raise important questions to be answered by future research. What leads to the individual differences on the FMA problems, if not the ability to control attention? Are these factors consistent with the automatic process view of restructuring? Do high and low attentional capacity individuals use fundamentally different cognitive processes to overcome the representational difficulties imposed by insight problems? Further research of this type, in which manipulations that isolate the stages of insight are refined and a larger variety of predictors of problem-solving performance are investigated, will clearly lead to a better understanding of the nature of restructuring in insight.

\section{REFERENCES}

AshCRAFT, M. H. (1994). Human memory and cognition (2nd ed.). New York: HarperCollins.

Baddeley, A. D., \& Hitch, G. J. (1974). Working memory. In G. H. Bower (Ed.), The psychology of learning and motivation (Vol. 8, pp. 47-90). New York: Academic Press.

Conway, A. R. A., \& Engle, R. W. (1994). Working memory and retrieval: A resource-dependent inhibition model. Journal of Experimental Psychology: General, 123, 354-373. 
Conway, A. R. A., \& Kane, M. J. (2001). Capacity, control, and conflict: An individual differences perspective on attentional capture. In C. L. Folk \& B. S. Gibson (Eds.), Attraction, distraction, and action: Multiple perspectives on attentional capture (pp. 349-372). Amsterdam: Elsevier.

Davidson, J. E. (1995). The suddenness of insight. In R. J. Sternberg \& J. E. Davidson (Eds.), The nature of insight (pp. 125-155). Cambridge, MA: MIT Press.

HAMBRICK, D. Z., \& ENGLE, R. W. (2003). The role of working memory in problem solving. In J. E. Davidson \& R. J. Sternberg (Eds.), The psychology of problem solving (pp. 176-206). Cambridge: Cambridge University Press.

HiтcH, G. J. (1978). The role of short-term working memory in mental arithmetic. Cognitive Psychology, 10, 302-323.

Kane, M. J., Bleckley, M. K., Conway, A. R. A., \& Engle, R. W. (2001). A controlled-attention view of working-memory capacity Journal of Experimental Psychology: General, 130, 169-183.

KANe, M. J., \& ENGLE, R. W. (2003). Working memory capacity and the control of attention: The contributions of goal neglect, response competition, and task set to Stroop interference. Journal of Experimental Psychology: General, 132, 47-70.

Kane, M. J., Hambrick, D. Z., Tuholski, S. W., Wilhelm, O., Payne, T. W., \& ENGLE, R. W. (2004). The generality of working memory capacity: A latent-variable approach to verbal and visuospatial memory span and reasoning. Journal of Experimental Psychology: General, 133, 189-217.

Kaplan, C. A., \& Simon, H. A. (1990). In search of insight. Cognitive Psychology, 22, 374-419.

Katona, G. (1940). Organizing and memorizing: Studies in the psychology of learning and teaching. New York: Columbia University Press.
KLINE, R. B. (1998). Principles and practice of structural equation modeling. New York: Guilford.

Knoblich, G., Ohlsson, S., Haider, H., \& Rhenius, D. (1999). Constraint relaxation and chunk decomposition in insight problem solving. Journal of Experimental Psychology: Learning, Memory, \& Cognition, 25, 1534-1555.

Knoblich, G., Ohlsson, S., \& Raney, G. E. (2001). An eye movement study of insight problem solving. Memory \& Cognition, 29, 1000-1009.

Metcalfe, J., \& Wiebe, D. (1987). Intuition in insight and noninsight problem solving. Memory \& Cognition, 15, 238-246.

Newell, A., \& Simon, H. A. (1972). Human problem solving. Englewood Cliffs, NJ: Prentice Hall.

OHLSSON, S. (1992). Information processing explanations of insight and related phenomena. In M. T. Keane \& K. J. Gilhooly (Eds.), Advances in the psychology of thinking (pp. 1-44). London: Harvester Wheatsheaf.

Ormerod, T. C., MacGregor, J. N., \& Chronicle, E. P. (2002). Dynamics and constraints in insight problem solving. Journal of Experimental Psychology: Learning, Memory, \& Cognition, 28, 791-799.

Schooler, J. W., OHLsson, S., \& Brooks, K. (1993). Thoughts beyond words: When language overshadows insight. Journal of Experimental Psychology: General, 122, 166-183.

Seifert, C. M., Meyer, D. E., Davidson, N., Patalano, A. L., \& Yaniv, I. (1995). Demystification of cognitive insight: Opportunistic assimilation and the prepared-mind perspective. In R. J. Sternberg \& J. E. Davidson (Eds.), The nature of insight (pp. 65-124). Cambridge, MA: MIT Press.

(Manuscript received June 23, 2004; revision accepted for publication May 17, 2005.) 